\title{
Short- and Long-Term Outcomes of Simultaneous Hepatic Artery Resection and Reconstruction for Perihilar Cholangiocarcinoma
}

\author{
Yoshitaro Shindo $^{\mathrm{a}}$ Shogo Kobayashib Hiroshi Wadab Yukio Tokumitsu ${ }^{\mathrm{a}}$

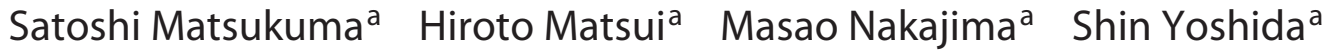 \\ Michihisa lida ${ }^{a}$ Nobuaki Suzuki ${ }^{a}$ Shigeru Takeda ${ }^{a}$ Yoshinobu Hoshiic \\ Hidetoshi Eguchi $^{\mathrm{b}}$ Hiroaki Nagano ${ }^{\mathrm{a}}$ \\ aDepartment of Gastroenterological, Breast and Endocrine Surgery, Yamaguchi University Graduate School of \\ Medicine, Ube, Japan; 'bepartment of Gastroenterological Surgery, Osaka University Graduate School of Medicine, \\ Osaka, Japan; 'Department of Diagnostic Pathology, Yamaguchi University Hospital, Ube, Japan
}

\section{Keywords \\ Perihilar cholangiocarcinoma · Hepatic artery resection · Hepatectomy}

\begin{abstract}
Introduction: Because surgical resection with simultaneous hepatic artery $(\mathrm{HA})$ resection and reconstruction for perihilar cholangiocarcinoma (PHC) is technically demanding, the surgical indication for this challenging procedure is controversial. Thus, this study aimed to evaluate the efficacy of simultaneous HA resection and reconstruction for PHC. Methods: Between January 2002 and January 2018, 13 patients with $\mathrm{PHC}$ underwent surgical intervention with simultaneous resection and reconstruction of the HA at Yamaguchi University Hospital (Ube, Japan) and Osaka University Hospital (Suita, Japan). Results: There were 2 cases (15.4\%) of 90day postoperative mortality. Nine patients (69.2\%) developed major postoperative complications (Clavien-Dindo classification $\geq \mathrm{Illa}$ ). Curative resections (R0) were achieved in 8 cases $(61.5 \%)$. The median survival time (MST) and 1- and 3-year survival rates after resection (including in-hospital deaths) were 20.9 months and 61.5 and $10.3 \%$, respectively. The MST and 1- and 2-year survival rates of 8 patients who
\end{abstract}

karger@karger.com www.karger.com/gat

Karger $\stackrel{\text { ' }}{5}$

BOPEN ACCESS
(C) 2020 The Author(s)

Published by S. Karger AG, Basel

This article is licensed under the Creative Commons AttributionNonCommercial-NoDerivatives 4.0 International License (CC BYNC-ND) (http://www.karger.com/Services/OpenAccessLicense). Usage and distribution for commercial purposes as well as any distribution of modified material requires written permission. underwent R0 resection were significantly better than those of the other 5 patients ( 24.2 vs. 10.2 months, 75.0 vs. $40.0 \%$, and 50.0 vs. $0.0 \%$, respectively, $p=0.0228$ ). Conclusions: Simultaneous $\mathrm{HA}$ resection and reconstruction is technically possible and may provide long-term survival in selected patients with locally advanced PHC.

(C) 2020 The Author(s)

Published by S. Karger AG, Basel

\section{Introduction}

Perihilar cholangiocarcinoma (PHC), which is a relatively rare tumor originating between the secondary branches of the right and left hepatic ducts and the common hepatic duct proximal to the cystic duct origin, is one of the most life-threatening malignancies [1]. Surgical resection is considered the only curative treatment for PHC because chemotherapy with or without radiation is less effective $[2,3]$. Moreover, complete resection with a negative margin is essential to improve long-term survival. PHC with vascular involvement remains a surgical challenge because this surgery is associated with high risks of postoperative morbidity and mortality. Although portal vein (PV) invasion was pre- 
viously considered unmanageable for $\mathrm{PHC}$, PV resection has been demonstrated to be a feasible and effective surgical option with acceptable morbidity, mortality, and survival outcome $[4,5]$. On the contrary, surgical resection with simultaneous hepatic artery (HA) resection and reconstruction for $\mathrm{PHC}$ is a technically demanding procedure [5-8]. Recently, living-donor liver transplantation has evolved as a usual and standard medical treatment, and HA resection and reconstruction for PHC has been technically accepted in Japan [9, $10]$.

To our knowledge, few studies have reported outcomes of HA resection and reconstruction for PHC. Therefore, the surgical indication for such a challenging procedure is controversial. Hence, in this study, we aimed to investigate the postoperative complications and survival outcomes in patients who underwent HA resection and reconstruction for PHC.

\section{Materials and Methods}

Between January 2002 and January 2018, consecutive PHC patients who underwent surgical resection with simultaneous resection and reconstruction of the HA at Yamaguchi University Hospital (Ube, Japan) and Osaka University Hospital (Suita, Japan) were identified. This study was approved by the Institutional Review Boards of Yamaguchi University Hospital (No. H30-062) and Osaka University Hospital (No. 18526). The study protocol was performed in accordance with the Declaration of Helsinki.

Preoperative computed tomography (CT) and/or magnetic resonance imaging were preferably performed before biliary drainage to evaluate the extent of tumor invasion since the images could be obscured by the inserted plastic or metallic stent. The surgical procedures, conducted based on the site of involved bile duct and residual liver function, were left trisectionectomy $(n=8)$, left hepatectomy with caudate lobectomy $(n=4)$, and right trisectionectomy $(n=1)$ (Table 1$)$. All patients underwent en bloc resection of the caudate lobe and extrahepatic bile duct. Indications for HA resection were determined by preoperative investigation with CT angiography and macroscopic inspection during surgery. HA resection was performed only when the vessels could not be detached from the tumor. HA reconstructions were conducted after the removal of specimens and before biliary reconstructions. HA was reconstructed with end-to-end anastomosis $(n=12)$ and an interposition autologous from left HA $(n=1)$ using interrupted 8-0 nonabsorbable monofilament. Doppler ultrasonography was performed immediately after the completion of the artery reconstruction. It was repeated twice daily to observe the arterial flow. One week after surgery, enhanced abdominal CT was performed to document a patent arterial anastomosis. Postoperative anticoagulant therapy was not administered to any of the patients.
Statistical Analysis

Categorical variables were compared using the $\chi^{2}$ test and Fisher's exact test. Survival curves were analyzed by the Kaplan-Meier method and the log-rank test. Statistical analyses were performed with GraphPad Prism V8.0 (GraphPad Software, Inc., San Diego, CA, USA). A $p$ value of $<0.05$ was considered statistically significant.

\section{Results}

The clinical and surgical characteristics of the 13 patients are summarized in Table 1 . Of these patients, 7 were men and 6 were women, with a median age of 66 years (range 57-78). Patient distributions by Bismuth classification were as follows: type IV in 10, type IIIb in 2 , and type IIIa in 1 patient [11]. Concomitant PV resection was performed in 6 patients. The median operative time was $731 \mathrm{~min}$ (range 625-1,091 min), and the median volume of blood loss was $1,700 \mathrm{~mL}$ (range $890-3,400 \mathrm{~mL}$ ). Curative resections (R0) were achieved in 8 cases $(61.5 \%)$. Of 13 patients, 5 patients (38.5\%) who underwent HA resection had histological positive tumor invasion of the resected artery. Clavien-Dindo III or higher postoperative complications occurred in 9 patients (69.2\%). The major complications were bile leakage $(n=5)$, bleeding $(n=3)$, and hepatic abscess $(n=1)$. There were 2 cases $(15.4 \%)$ of 90 -day postoperative mortality. The cause of death was postoperative bleeding. Although no patients underwent neoadjuvant chemotherapy, 4 underwent postoperative adjuvant chemotherapy, comprising gemcitabine in 3 patients and S- 1 in 1 patient.

Surgical outcomes, morbidity, and mortality were compared between the earlier (2002-2010) and later periods (2011-2018) (Table 2). Although operative time, blood loss, and morbidity were similar in the 2 periods, all procedures performed in the later period involved difficult and intricate left trisectionectomy. Nevertheless, the mortality rate was $25 \%$ in the earlier period, while there no deaths in the later period.

Postoperative recurrence was found in 9 patients (69.2\%), including local recurrence $(n=4)$, lymph node metastasis $(n=2)$, liver metastasis $(n=2)$, and peritoneal dissemination $(n=1)$. R1 resection was not associated with the site of 1 st recurrence. The median survival time (MST) and 1- and 3-year survival rates after resection (including in-hospital deaths) were 20.9 months and 61.5 and $10.3 \%$, respectively. The MST and 1- and 2-year survival rates of 8 patients who underwent $\mathrm{R} 0$ resection were significantly better than those of the other 5 patients $(24.2$ vs. 10.2 months, 75.0 vs. $40.0 \%$, and 50.0 vs. $0.0 \%$, respec- 


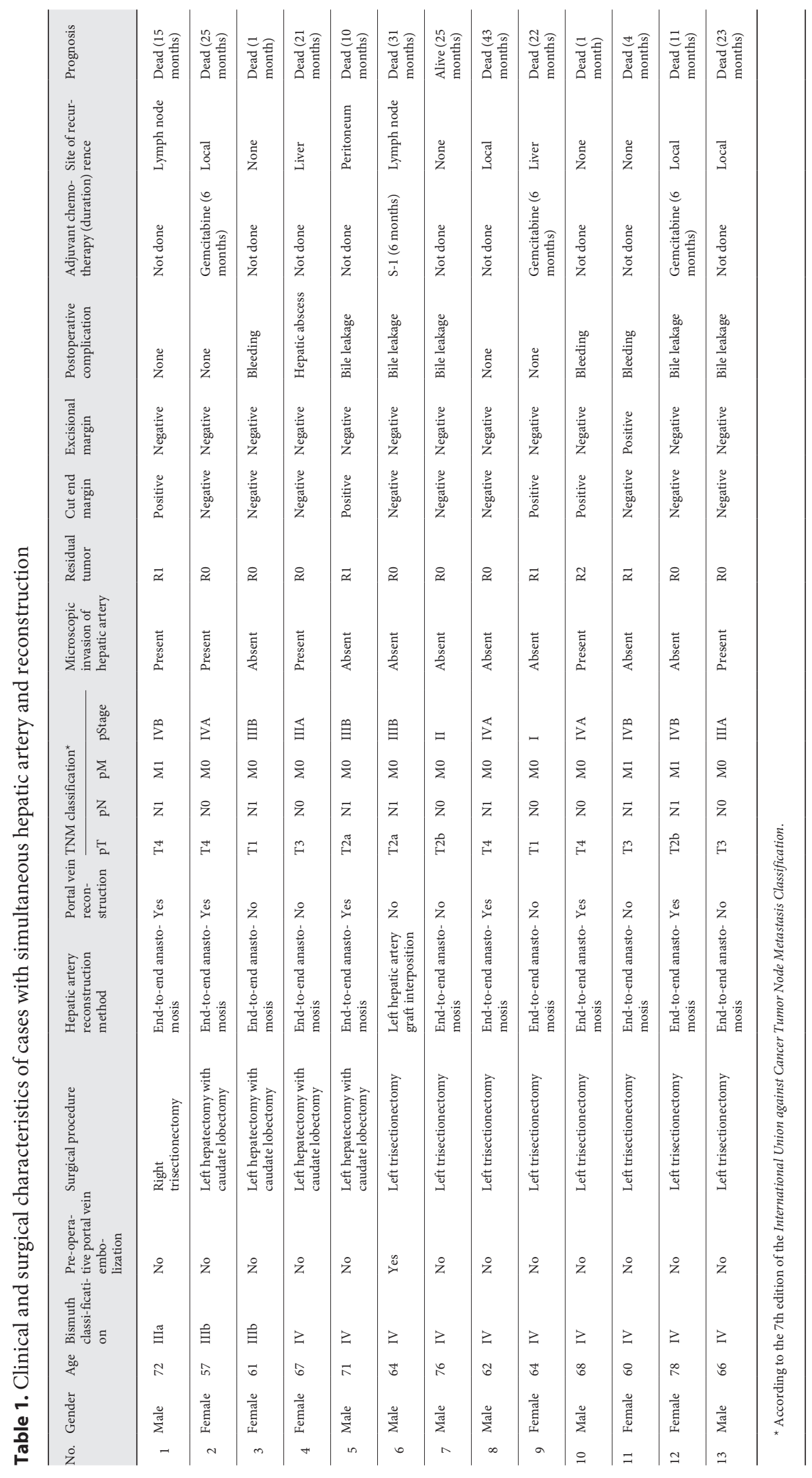


Table 2. Surgical outcomes, morbidity, and mortality according to the time period

\begin{tabular}{|c|c|c|c|}
\hline Variable & $\begin{array}{l}\text { Earlier period } \\
(2002-2010)\end{array}$ & $\begin{array}{l}\text { Later period } \\
(2011-2018)\end{array}$ & $p$ value \\
\hline Patients, $n$ & 8 & 5 & \\
\hline Age & $66(57-72)$ & $66(60-78)$ & 0.5501 \\
\hline \multicolumn{4}{|l|}{ Gender } \\
\hline Male & 4 & 3 & $>0.9999$ \\
\hline Female & 4 & 2 & \\
\hline \multicolumn{4}{|l|}{ Surgical procedure } \\
\hline Left hepatectomy with caudate lobectomy & 4 & 0 & 0.1049 \\
\hline Right or left trisectionectomy & 4 & 5 & \\
\hline Portal vein resection & 5 & 1 & 0.2657 \\
\hline Operative time, $\min$ & $743(625-1,091)$ & $727(625-811)$ & 0.5501 \\
\hline Intraoperative blood loss, $\mathrm{mL}$ & $2,161(890-3,400)$ & $1,890(900-1,700)$ & 0.1709 \\
\hline Morbidity (CD >IIIa), $n(\%)$ & $4(50)$ & $5(100)$ & 0.1049 \\
\hline Bile leakage & 1 & 4 & \\
\hline Hepatic abscess & 1 & 0 & \\
\hline Bleeding & 2 & 1 & \\
\hline Mortality, $n(\%)$ & $2(25)$ & $0(0)$ & 0.4872 \\
\hline
\end{tabular}

Values are represented as median (range). CD, Clavien-Dindo classification.

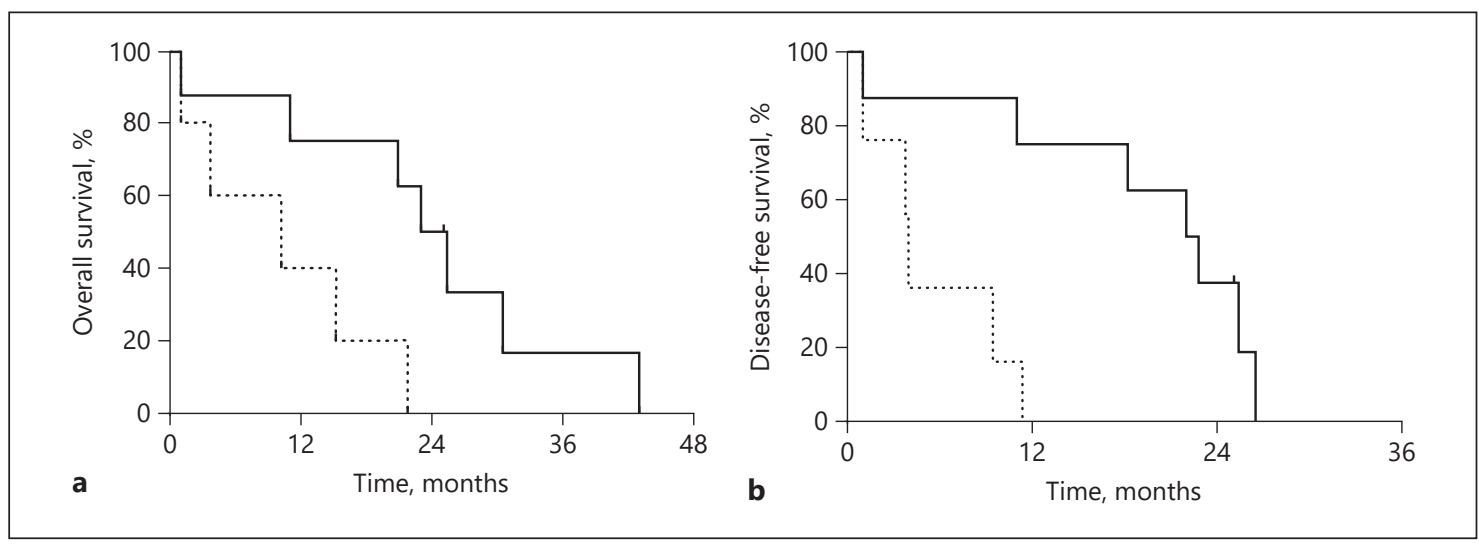

Fig. 1. a Overall survival rates of patients with negative surgical margin $(n=8$, solid line) and with positive surgical margins $(n=5$, dotted line). A significant difference is observed between groups $(p=0.0228)$. b Disease-free survival rates of patients with negative surgical margin $(n=8$, solid line) and with positive surgical margins ( $n=5$, dotted line). A significant difference is observed between groups $(p=0.0065)$.

tively; $p=0.0228$ ) (Fig. 1a). The MST and 1-year diseasefree survival rates of 8 patients who underwent $\mathrm{R} 0$ resection were also significantly better than those of the other 5 patients ( 22.4 vs. 3.7 months and 75.0 vs. $0.0 \%$, respectively; $p=0.0065$ ) (Fig. 1b).

Preoperative and intraoperative factors were compared between patients undergoing $\mathrm{R} 0$ and $\mathrm{R} 1$ resections (Table 3). There were no significant differences in preoperative biliary drainage, Bismuth classification, preopera- tive tumor markers, tumor size, surgical procedures, operative time, or blood loss.

\section{Discussion}

This study focused on the surgical and survival outcomes of HA resection and reconstruction for PHC, based on 13 cases. We performed left-sided hepatectomy 
Table 3. Comparison of preoperative and intraoperative factors between the R 0 and $\mathrm{R} 1$ resection patients

\begin{tabular}{lccc}
\hline Variable & R0 resection $(n=8)$ & R1 resection $(n=4)$ & $p$ value \\
\hline Age & $65(57-78)$ & $67(60-72)$ & 0.9677 \\
Gender & 4 & 2 & $>0.9999$ \\
$\quad$ Male & 4 & 2 & 0.5152 \\
$\quad$ Female & 6 & 4 & $>0.9999$ \\
Preoperative biliary drainage & & 1 & 0.8081 \\
Bismuth classification & 2 & 3 & 0.7111 \\
$\quad$ IIIa or IIIb & 6 & $23.5(15-1,558)$ & $>0.9999$ \\
$\quad$ IV & $52.5(3.4-1,817)$ & $22(10-55)$ & $>0.9999$ \\
Preoperative CA19-9, U/mL & $28(12-50)$ & 2 & 0.4869 \\
Tumor size, mm & 3 & 2 & 0.6828 \\
Surgical procedure & 5 & 2 & \\
$\quad$ Left hepatectomy with caudate lobectomy & 3 & $686(625-762)$ & \\
$\quad$ Right or left trisectionectomy & $733(625-885)$ & $1,470(900-2,450)$ & \\
Portal vein resection & $1,786(890-3,300)$ & & \\
Operative time, min & \multicolumn{3}{c}{} \\
Intraoperative blood loss, mL & 5 & & \\
\hline
\end{tabular}

Values are represented as median (range).

in 12 patients $(92.3 \%)$. Because the right HA, mainly located behind the biliary confluence, is often involved in tumor expansion, right $\mathrm{HA}$ resection and reconstruction is needed in left-sided liver resection for PHC $[6,8]$. HA resection is less common, and the indication of this challenging procedure is controversial. Some authors have reported that HA resection for PHC was not justified because of high surgical morbidity and mortality rates $(10.0-55.6 \%)$ with no long-term survivors $[5,6,12]$. Recent advances in operative techniques and perioperative management for HA resection and reconstruction with introduction and generalization of living-donor liver transplantation have decreased the morbidity and mortality rates (3.6-4.2\%) [13-15]. Although 2 deaths (15.4\%) were recorded in this study, there was no mortality in 5 patients over the last decade.

Postoperative cancer prognosis with concomitant PHC and HA involvement should be considered when determining the indication for HA resection and reconstruction. Gemcitabine with cisplatin combination is the standard therapy of unresectable PHC based on a phase III randomized controlled trial (ABC-02 trial) [16]. Although this combination chemotherapy led to longer survival than gemcitabine alone, the MST and 3-year survival rate was 11.7 months and nearly $0 \%$, respectively [16]. In contrast, a study reported that the 1-, 3-, and 5-year survival rates in $50 \mathrm{PHC}$ patients with simultaneous resections and reconstructions of PV and HA were 78.9, 36.3, and 30.3\%, respectively, with only $2 \%$ mortality [8]. Therefore, surgical resection is the only potentially curative treatment for PHC. R0 resection is considered the prognostic factor for long-term survival in PHC patients after surgery [17]. Indeed, patients who underwent $\mathrm{R} 0$ resection had better prognosis than other patients in this study $(p=0.0228)$ (Fig. 1a). Because patients without R0 resection have a poor prognosis, artery resection should be performed only for selected patients who could achieve negative resection margins.

It is important to preoperatively diagnose tumor invasion of the HA. Artery involvement on imaging may not coincide with intraoperative or histological findings because $18-54 \%$ of the patients who underwent HA resection had histological positive tumor invasion $[5,8,15]$. In this study, histological positive tumor invasion of the resected HA was observed in 5 of 13 patients (38.5\%). Although case \#7 showed no histological involvement of the right $\mathrm{HA}$, carcinoma cells surrounded the right HA with a distance of $230 \mu \mathrm{m}$ between the tunica media of the right $\mathrm{HA}$ and the leading edge of carcinoma cells (Fig. 2). If the right HA had been preserved, the surgical margin would be positive. Therefore, HA resection was needed to achieve negative margins. These results indicate that the indication for artery resection should be made based on intraoperative as well as preoperative findings.

In this study, curative resection with negative margin has been carried out in 8 patients $(61.5 \%)$, which might 

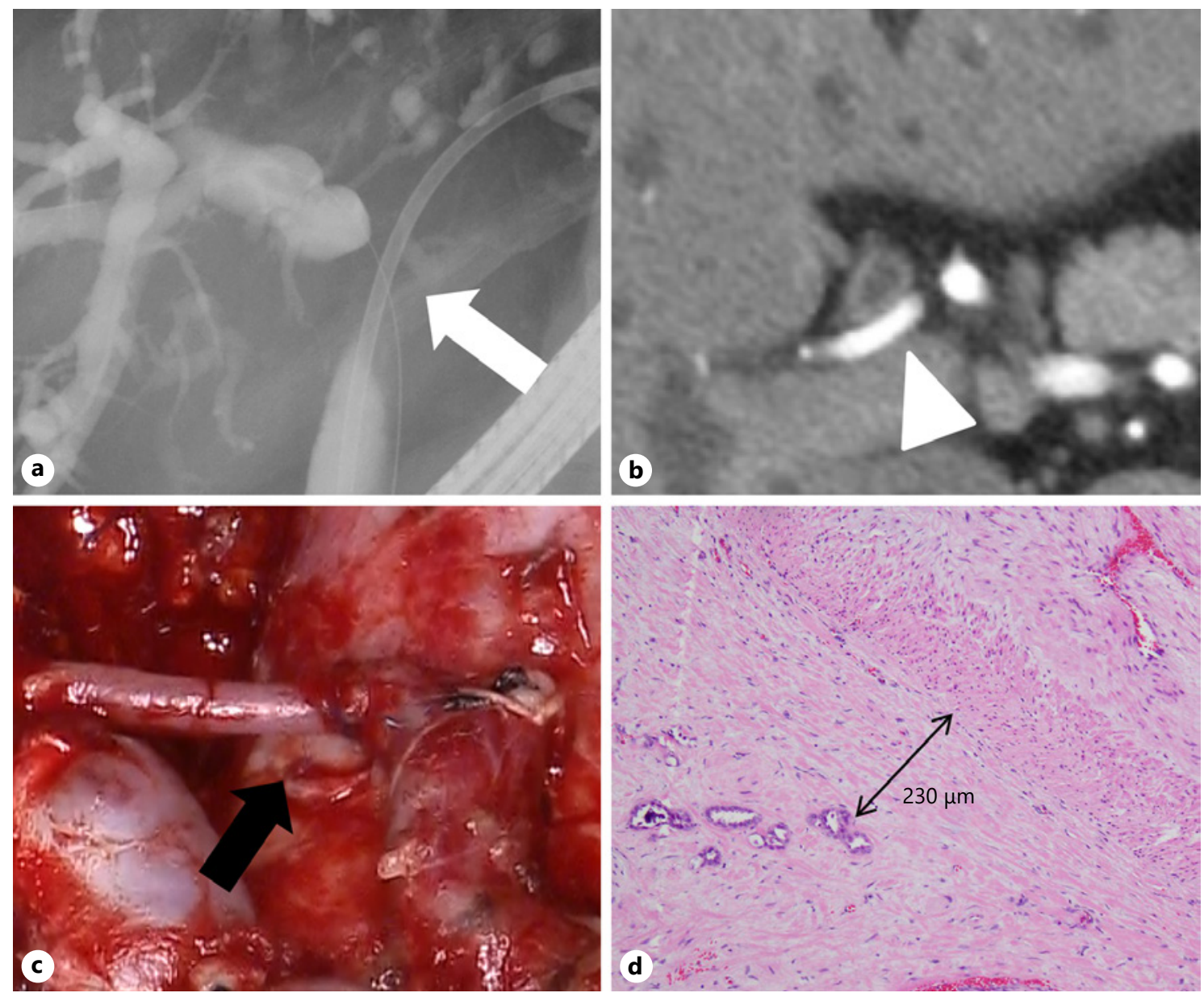

Fig. 2. Preoperative, intraoperative, and histological images of case \#7 in Table 1. a Endoscopic retrograde cholangiography showed a Bismuth type IV tumor. b Enhanced computed tomography demonstrated abutment of the right hepatic artery (arrow head). c The right hepatic artery was reconstructed with end-to-end anastomosis. d The right hepatic artery was not involved histologically, although carcinoma cells were observed to surround the right hepatic artery. The distance between the tunica media of the right hepatic artery and the leading edge of carcinoma cells was $230 \mu \mathrm{m}$.

indicate that most PHC had aggressive local invasion. Currently, surgery alone is considered the standard treatment for PHC. However, patients undergoing curative resection mostly develop recurrent tumor disease. Of the 8 patients with R0 resection, recurrence was seen in 6 patients (75.0\%). The most common recurrence type was local recurrence $(n=4)$, followed by lymph node metastasis $(n=1)$ and liver metastasis $(n=1)$. Surgery alone is not a sufficient therapy for this aggressive tumor because the prognosis of PHC remains poor. Although recent randomized phase III studies of adjuvant therapy such as capecitabine, gemcitabine, and oxaliplatin were conducted to improve survival probability, they did not demonstrate a survival benefit for biliary tract cancers including
PHC [18-20]. Two randomized phase III trials of adjuvant therapy are currently ongoing. One trial from Japan Clinical Oncology Group compares the effect of adjuvant S-1, an oral tegafur-based fluoropyrimidine, with that of surgery alone [21]. The other ACTICCA-1 trial from Europe evaluated the efficacy of gemcitabine and cisplatin [22]. Although no patients received neoadjuvant therapy in this study, neoadjuvant therapy such as chemotherapy and chemoradiotherapy seems to be another treatment option with the rationale to downstage and downsize the tumor to enable R0 resection and eliminate microscopic distant metastases [23-25]. Up to now, although no evidence is established on the effectiveness of neoadjuvant and adjuvant treatment in PHC, future studies of multi- 
modality therapy would be expected to develop novel treatment strategies. This study has a number of limitations, including a small sample size and retrospective analysis. Further multicenter studies with more patients are needed to confirm these results. In conclusion, simultaneous HA resection and reconstruction is technically possible and may provide long-term survival in selected patients with locally advanced PHC.

\section{Acknowledgements}

The authors would like to thank Dr. Masahiro Tanabe, Seiji Kaino, and Tatsuya Ioka for useful discussions.

\section{Statement of Ethics}

This study was approved by the Institutional Review Boards of Yamaguchi University Hospital (No. H30-062) and Osaka University Hospital (No. 18526). The study protocol was performed in accordance with the Declaration of Helsinki, and all patients provided written informed consent.

\section{Conflict of Interest Statement}

The authors have no conflicts of interest to declare.

\section{Funding Sources}

The authors did not receive any funding.

\section{Author Contributions}

Yoshitaro Shindo, Shogo Kobayashi, and Hiroaki Nagano designed the study. Shogo Kobayashi, Hiroshi Wada, Yukio Tokumitsu, Satoshi Matsukuma, Hiroto Matsui, Masao Nakajima, and Shin Yoshida contributed to acquisition of data. Yoshitaro Shindo, Michihisa Iida, and Yoshinobu Hoshii conducted data analysis and interpretation. Yoshitaro Shindo, Nobuaki Suzuki, and Shigeru Takeda drafted the manuscript. Hidetoshi Eguchi and Hiroaki Nagano reviewed the statistics and contributed to critical revisions. All authors have approved the final version of the article.

\section{References}

1 Rassam F, Roos E, van Lienden KP, van Hooft JE, Klümpen HJ, van Tienhoven G, et al. Modern work-up and extended resection in perihilar cholangiocarcinoma: the AMC experience. Langenbecks Arch Surg. 2018; 403(3):289-307.

2 Ebata T, Ercolani G, Alvaro D, Ribero D, Di Tommaso L, Valle JW. Current status on cholangiocarcinoma and gallbladder cancer. Liver Cancer. 2016;6(1):59-65.

3 Matsukuma S, Tokumitsu Y, Shindo Y, Matsui $H$, Nagano $H$. Essential updates to the surgical treatment of biliary tract cancer. Ann Gastroenterol Surg. 2019;3(4):378-89.

4 Ebata T, Nagino M, Kamiya J, Uesaka K, Nagasaka T, Nimura Y. Hepatectomy with portal vein resection for hilar cholangiocarcinoma: audit of 52 consecutive cases. Ann Surg. 2003; 238(5):720-7.

5 Miyazaki M, Kato A, Ito H, Kimura F, Shimizu $\mathrm{H}$, Ohtsuka $\mathrm{M}$, et al. Combined vascular resection in operative resection for hilar cholangiocarcinoma: does it work or not? Surgery. 2007;141(5):581-8.

6 Yamanaka N, Yasui C, Yamanaka J, Ando T, Kuroda N, Maeda S, et al. Left hemihepatectomy with microsurgical reconstruction of the right-sided hepatic vasculature. A strategy for preserving hepatic function in patients with proximal bile duct cancer. Langenbecks Arch Surg. 2001;386(5):364-8.
7 Sakamoto Y, Sano T, Shimada K, Kosuge T, Kimata Y, Sakuraba M, et al. Clinical significance of reconstruction of the right hepatic artery for biliary malignancy. Langenbecks Arch Surg. 2006;391(3):203-8.

8 Nagino $M$, Nimura $Y$, Nishio $H$, Ebata T, Igami T, Matsushita M, et al. Hepatectomy with simultaneous resection of the portal vein and hepatic artery for advanced perihilar cholangiocarcinoma: an audit of 50 consecutive cases. Ann Surg. 2010;252(1):115-23.

9 Marubashi S, Kobayashi S, Wada H, Kawamoto K, Eguchi H, Doki Y, et al. Hepatic artery reconstruction in living donor liver transplantation: risk factor analysis of complication and a role of MDCT scan for detecting anastomotic stricture. World J Surg. 2013; 37(11):2671-7

10 Ikegami T, Hashikura Y, Nakazawa Y, Urata $\mathrm{K}$, Mita A, Ohno Y, et al. Risk factors contributing to hepatic artery thrombosis following living-donor liver transplantation. J Hepatobiliary Pancreat Surg. 2006;13(2):105-9.

11 Bismuth H, Nakache R, Diamond T. Management strategies in resection for hilar cholangiocarcinoma. Ann Surg. 1992;215(1):31-8.

12 Gerhards MF, van Gulik TM, de Wit LT, Obertop H, Gouma DJ. Evaluation of morbidity and mortality after resection for hilar cholangiocarcinoma: a single center experience. Surgery. 2000;127(4):395-404.
13 Wang ST, Shen SL, Peng BG, Hua YP, Chen $B$, Kuang M, et al. Combined vascular resection and analysis of prognostic factors for hilar cholangiocarcinoma. Hepatobiliary \& pancreatic diseases international. HBPD Int 2015;14(6):626-32.

14 Nagino M, Ebata T, Yokoyama Y, Igami T, Sugawara G, Takahashi Y, et al. Evolution of surgical treatment for perihilar cholangiocarcinoma: a single-center 34-year review of 574 consecutive resections. Ann Surg. 2013; 258(1):129-40.

15 Noji T, Tsuchikawa T, Okamura K, Tanaka K, Nakanishi Y, Asano T, et al. Concomitant hepatic artery resection for advanced perihilar cholangiocarcinoma: a case-control study with propensity score matching. J Hepatobiliary Pancreat Sci. 2016;23(7):442-8.

16 Valle J, Wasan H, Palmer DH, Cunningham D, Anthoney A, Maraveyas A, et al. Cisplatin plus gemcitabine versus gemcitabine for biliary tract cancer. N Engl J Med. 2010;362(14): 1273-81.

17 Nagino M. Fifty-year history of biliary surgery. Ann Gastroenterol Surg. 2019;3(6):598605

18 Primrose JN, Fox RP, Palmer DH, Malik HZ Prasad R, Mirza D, et al. Capecitabine compared with observation in resected biliary tract cancer (BILCAP): a randomised, controlled, multicentre, phase 3 study. Lancet Oncol. 2019;20(5):663-73. 
19 Edeline J, Benabdelghani M, Bertaut A, Watelet J, Hammel P, Joly J-P, et al. Gemcitabine and oxaliplatin chemotherapy or surveillance in resected biliary tract cancer (PRODIGE 12-ACCORD 18-UNICANCER GI): a randomized phase III study. J Clin Oncol. 2019; 37(8):658-67.

20 Ebata T, Hirano S, Konishi M, Uesaka K, Tsuchiya Y, Ohtsuka M, et al. Randomized clinical trial of adjuvant gemcitabine chemotherapy versus observation in resected bile duct cancer. Br J Surg. 2018;105(3):192-202.

21 Nakachi K, Konishi M, Ikeda M, Mizusawa J, Eba J, Okusaka T, et al. A randomized phase III trial of adjuvant S-1 therapy vs. observa- tion alone in resected biliary tract cancer: Japan Clinical Oncology Group Study (JCOG1202, ASCOT). Jpn J Clin Oncol. 2018; 48(4):392-5.

22 Stein A, Arnold D, Bridgewater J, Goldstein D, Jensen LH, Klümpen HJ, et al. Adjuvant chemotherapy with gemcitabine and cisplatin compared to observation after curative intent resection of cholangiocarcinoma and muscle invasive gallbladder carcinoma (ACTICCA-1 trial): a randomized, multidisciplinary, multinational phase III trial. BMC Cancer. 2015;15: 564.

23 Kobayashi S, Tomokuni A, Gotoh K, Takahashi H, Akita H, Marubashi S, et al. A retro- spective analysis of the clinical effects of neoadjuvant combination therapy with full-dose gemcitabine and radiation therapy in patients with biliary tract cancer. Eur J Surg Oncol. 2017;43(4):763-71.

24 Frosio F, Mocchegiani F, Conte G, Bona ED, Vecchi A, Nicolini D, et al. Neoadjuvant therapy in the treatment of hilar cholangiocarcinoma: review of the literature. World J Gastrointest Surg. 2019;11(6):279-86.

25 Baltatzis M, Jegatheeswaran S, Siriwardena AK. Neoadjuvant chemoradiotherapy before resection of perihilar cholangiocarcinoma: a systematic review. Hepatobiliary Pancreat Dis Int. 2020;19(2):103-8. 